\title{
Experimental Measurements of U60 Nanocluster Stability in Aqueous Solution
}

\section{Shannon L. Flynn ${ }^{1} *$, Jennifer E.S. Szymanowski ${ }^{1,}$, Yunyi Gao ${ }^{2,}$, Tianbo Liu ${ }^{2,}$, Peter C. Burns $^{1,}$ and Jeremy B. Fein ${ }^{1,}$}

\section{Department of Civil and Environmental Engineering and Earth Sciences,} University of Notre Dame, Notre Dame, IN 46556, USA

2. Department of Polymer Science, The University of Akron, Akron, $\mathrm{OH} 44325, \mathrm{USA}$

* Corresponding Author: Shannon.L.Flynn.104@nd.edu

\section{Abstract}

In this study, the aqueous behavior of isolated U60 nanoclusters $\left(\mathrm{K}_{16} \mathrm{Li}_{25}\left[\mathrm{UO}_{2}\left(\mathrm{O}_{2}\right) \mathrm{OH}\right]_{60}\right)^{-19}$ was studied under several $\mathrm{pH}$ conditions and nanocluster concentrations to determine if the nanoclusters exhibit solid phase buffering behavior or if they exhibit behavior more like aqueous complexes. $\mathrm{U} 60$ is a cage cluster consisting of $60\left(\mathrm{UO}_{2}\right)\left(\mathrm{O}_{2}\right)_{2}(\mathrm{OH})_{2}$ uranyl polyhedral which share $\mathrm{OH}$ and $\mathrm{O}_{2}$ groups with their neighboring uranyl polyhedral, resulting in negatively charged cage clusters whose charge is at least partially offset by $\mathrm{K}^{+}$and $\mathrm{Li}^{+}$in the aqueous phase. Batch experiments to monitor nanocluster stability were conducted for 16 days at $\mathrm{pH} 7.5,8.0$ and 8.5 at nanocluster suspension concentrations of $1.4,2.8$ and $6.0 \mathrm{~g} / \mathrm{L}$. The aqueous concentrations of $\mathrm{U}, \mathrm{Li}$, and $\mathrm{K}$, determined after $10 \mathrm{~K} \mathrm{Da}$ molecular weight filtration, achieved steady-state with the nanoclusters within 24 hours. The steady-state aqueous $\mathrm{U}, \mathrm{Li}$, and $\mathrm{K}$ concentrations were independent of solution $\mathrm{pH}$, however they increased with increasing nanocluster concentration, indicating that the nanoclusters do not buffer the aqueous activities as a bulk solid phase would, but exhibit behavior that is more characteristic of dissolved aqueous complexes. The ion activity product (I.A.P.) value was calculated using two approaches: 1) treating the nanoclusters as a solid phase with an activity of one, and 2) treating the nanoclusters as aqueous complexes with a non-unit activity equal to their concentration in solution. The I.A.P. values that were calculated with non-unit activity for the nanoclusters exhibited significantly less variation as a function of nanocluster concentration compared to the I.A.P. values calculated with a nanocluster activity of one. The results yield a calculated log dissociation constant for the U60 nanoclusters of 9.2 $+0.2 /-0.3(1 \sigma)$. Our findings provide a better understanding of the thermodynamic stability and behavior of U60 nanoclusters in aqueous systems, and can be used to estimate the dissociation behavior of nanoclusters under a range of aqueous conditions.

\section{Introduction}

Uranyl peroxide nanoclusters represent a newly-discovered class of uranium compounds (Burns et al., 2005) whose presence in aqueous solution could dramatically alter the mobility and partitioning of uranium in environmental systems and industrial applications. While uranyl peroxide nanoclusters have not yet been observed to form in natural systems, they have the potential to form at contaminated sites, such as Hanford, Savannah River and Fukushima (Burns et al., 2012). The nanoclusters are uranyl peroxide spherical cages whose topologies resemble 
fullerenes and traditional polyoxometalate (POM) clusters (Sigmon et al., 2009b). The uranyl peroxide nanoclusters that have been described to date self-assemble in aqueous solution under ambient conditions and form crystals composed of nanocluster building blocks when the solution is allowed to evaporate. The nanoclusters within the crystals consist of uranyl bipyramids linked with hydroxide and peroxide bonded with the uranyl ions to form bridges, with the required curvature ascribed to a partially covalent interaction between the uranyl ion and a peroxide group (Vlaisavljevich et al., 2010). The uranyl peroxide cage alone has a net negative charge, but charge neutrality within the crystals is provided by the presence of various cations, depending on the cluster. To date, a range of structures of crystalline nanocluster materials have been described, but the aqueous stabilities of the constituent nanoclusters is unknown (Burns, 2011; Nyman and Burns, 2012; Qui and Burns, 2013).

The solubility of other solids that are composed of nanoclusters, such as transition metal oxide POM salts, have been studied extensively. Generally, POM salts of Mo, V and W are highly soluble whereas the salts of $\mathrm{Nb}$ and Mo are less soluble. The solubility of Mo, $\mathrm{V}$ and $\mathrm{W}$ POMs are related to the hydration radius of the ion pairing cations, with $\mathrm{Li}, \mathrm{Na}$ and $\mathrm{K}$ salts being the most soluble and $\mathrm{Cs}$ and $\mathrm{Rb}$ salts being the least soluble (Miro and Bo, 2012; Nyman and Burns, 2012: refs). The solubility of $\mathrm{Nb}$ and Ta POMs follow the inverse trend regarding cations with $\mathrm{Rb}$ and Cs salts being the most soluble (Antonio et al., 2009; Hou et al., 2012; Hou et al. 20011). To date, the crystals of the uranyl peroxide class nanoclusters are known to exhibit a high and undefined aqueous solubility predicted to follow a $\mathrm{Li}>\mathrm{Na}>\mathrm{K}>\mathrm{Cs}>\mathrm{Rb}$ trend (Nyman and Burns, 2012). Studies of nanoclusters and POMs in solution have largely focused on cation exchange behavior, self-assembly and the formation of stable assemblies, such as blackberries (Liu, 2002; Liu et al., 2003; Pigga et al., 2010; refs). The stability of uranyl peroxide nanoclusters in an aqueous solution as a function of time, $\mathrm{pH}$ and solution chemistry is still unknown.

Uranyl peroxide nanocluster crystals exhibit high solubilities in general, and when placed in undersaturated solutions, they dissolve completely and quickly, liberating distinct, isolated nanoclusters into solution (Qui et al., 2012). The diameter of the U60 $\left(\mathrm{K}_{16} \mathrm{Li}_{26}\left[\mathrm{UO}_{2}\left(\mathrm{O}_{2}\right) \mathrm{OH}\right]_{60}\right)$ cage cluster, which is the subject of this study, as measured from the outer edges of the bounding $\mathrm{O}$ atoms in the crystalline state, is $2.4 \mathrm{~nm}$ (Sigmon et al., 2009a), which is comparable in size to the larger molybdenum-oxide POMs (2.0 - $2.3 \mathrm{~nm}$ ) (Muller et al., 2001). There is qualitative evidence that isolated U60 nanoclusters remain intact in solution for at least several months (Armstrong et al., 2012). Although the structures of about 40 uranyl peroxide nanocluster crystals have been described (Burns, 2011), the nature and behavior of these nanoclusters in aqueous solution remains largely unexplored. If these types of nanoclusters form in Ucontaminated geologic systems, then their aqueous stability is a likely to be one of the factors controlling the fate and transport of uranium in these systems. Applications of uranyl peroxide nanoclusters in an advanced nuclear energy system, such as in fuel reprocessing, also requires an understanding of their behavior in solution.

Isolated nanoclusters in solution represent one of three possible $\mathrm{U}$ components in a nanoclusterwater system (Figure 1). Although neither of the reactions depicted in Figure 1 have been studied, both the reaction between isolated nanoclusters in solution and their parent crystals, and the reaction between isolated nanoclusters in solution and aqueous monomeric species, can be described as equilibrium reactions. The isolated nanocluster in solution represents a hybrid type 
of species. They are much larger than a typical aqueous species and they exhibit a regular and complex structure so they may exhibit some solid phase behaviors. However, they also have a limited size, with a fixed number of atoms in each cluster, so they have similarities with aqueous complexes. The primary objective of this research is to determine the aqueous stability of isolated U60 nanoclusters, and to determine if their aqueous behavior is that of a bulk solid phase or of large aqueous complexes. If an equilibrium exists between the nanoclusters and aqueous species and the nanoclusters behave as a bulk solid phase, then the activity product of aqueous species in equilibrium with the nanoclusters would be independent of nanocluster concentration in suspension. This behavior would be similar to the concentration-independent solubility that is observed for macroscopic solid phases. Conversely, if the nanoclusters behave as aqueous complexes, then an increase in the concentration of nanoclusters in solution would result in a concomitant increase in the activity product of aqueous species in equilibrium with them. Clearly, the fundamental properties of the hydrated nanoclusters must be determined in order to model their behavior and the mobility of uranium in systems in which nanoclusters are present.

In this study, we determined the reaction between isolated U60 nanoclusters in solution with its constituent aqueous species and the nature of this reaction. We tested whether an equilibrium or steady-state exists between the nanoclusters and aqueous monomeric species in order to determine whether or not the nanoclusters act as a bulk solid phase by buffering the concentration of the constituent aqueous species or if the nanoclusters behave as large aqueous complexes whose concentration in the system affects the activities of the constituent aqueous monomeric species. Experiments were conducted with U60 because its structure is wellcharacterized (Sigmon et al., 2009a), and pure crystals can be synthesized readily. We measured the concentration of U60 nanoclusters and their aqueous constituent monomeric species as a function of time, $\mathrm{pH}$ and nanocluster concentration. In the experimental systems, equilibrium could only be approached from under-saturation with respect to the U60 nanoclusters, as a pure yield of isolated U60 nanoclusters have yet to be synthesized from a solution of their constituent aqueous species (rather, the solution is polydisperse). We used the measured $\mathrm{pH}$ and aqueous $\mathrm{U}$, $\mathrm{Li}$, and $\mathrm{K}$ concentrations to calculate the ion activity product (I.A.P.) for each of the experimental conditions. We used the behavior of the calculated I.A.P. values as a function of $\mathrm{pH}$ and nanocluster concentration to define the nature of the nanoclusters in solution.

\section{2. Methods and Materials}

\subsection{Synthesis and Characterization of Nanoclusters}

114

115

116

117

118

119

120

121

U60 crystals were synthesized using methods modified from Sigmon et al. (2009b). In summary, the following compounds were added to a $20 \mathrm{~mL}$ uncapped glass vial: $1 \mathrm{~mL}$ of $0.436 \mathrm{M}$ uranyl nitrate hexahydrate, $0.25 \mathrm{~mL}$ of $0.285 \mathrm{M}$ potassium chloride, $1 \mathrm{~mL}$ of $30 \%$ hydrogen peroxide, and $0.75 \mathrm{ml}$ of $2.203 \mathrm{M}$ lithium hydroxide. The initial $\mathrm{pH}$ of the mother solution was approximately 9. After 7 days at room temperature, large, yellow, bipyramidal crystals had precipitated from solution. Crystals were harvested by vacuum filtration using a Whatman cellulose filter with an $11 \mu \mathrm{m}$ pore size and were rinsed with approximately $5 \mathrm{~mL}$ of $18 \mathrm{M} \Omega$ ultrapure water. 
The identity of the U60 crystals for each experiment was verified using single crystal X-ray diffractometry. A rinsed crystal was placed on a cryoloop in Infinium oil, and cooled to $110 \mathrm{~K}$ for data collection using a Bruker APEX diffractometer equipped with Mo $K \alpha$ radiation. A complete sphere of data was collected for each crystal using four sets of exposures with framewidths of $0.3^{\circ}$ in $\omega$. Data were corrected for Lorentz, polarization, and background effects using SADABS. Structure solutions and refinements were conducted with SHELXTL (Sheldrick, 1997).

Aggregation and agglomeration behavior of the U60 nanoclusters in solution was determined by measuring particle size using a Malvern Zetasizer Nano S, equipped with a He-Ne laser with a back scatter detector. Particle size was determined using the intensity of Rayleigh scattering. Each sample was analyzed with three sets of measurements composed of 10 replicate scans that were 30 seconds in length.

\subsection{Aqueous Partitioning Experiments}

The partitioning of $\mathrm{U}, \mathrm{Li}$, and $\mathrm{K}$ between the isolated $\mathrm{U} 60$ nanoclusters in solution and aqueous monomeric species was measured in batch experiments that were conducted at room temperature in Teflon reaction vessels. For each experiment, a known mass of synthesized U60 crystals (approximately 2-10 $\mathrm{mM}$ in diameter) was added to a known mass of $18 \mathrm{M} \Omega$ ultrapure water to achieve nanocluster suspension concentrations of approximately $1.4,2.8$ or $6.0 \mathrm{~g} / \mathrm{L}$. Each of these concentrations were low enough for the complete dissolution of the U60 crystals to be achieved, creating suspensions of isolated U60 nanoclusters that could further dissolve or dissociate to yield aqueous monomeric U, Li, and K species. Complete dissolution of the U60 crystal was confirmed in control experiments that measured no difference in total $\mathrm{U}$ concentrations in solutions before and after filtration through using $0.2 \mu \mathrm{m}$ PTFE membranes (Table S1). For each nanocluster concentration, experiments were conducted at three pHs: 7.5, 8.0 and 8.5. Duplicate and in one case triplicate experiments were conducted for the 9 experimental $\mathrm{pH} /$ nanocluster concentration conditions studied (Table $\mathrm{S} 2$ shows each of the experimental conditions). After dissolution of the U60 crystals, the $\mathrm{pH}$ of each experimental solution was adjusted through the gravimetric addition of $6 \mathrm{M} \mathrm{NaOH}$ and/or $\mathrm{HNO}_{3}$. The total mass of additions was recorded in order to calculate the ionic strength and solution speciation for each experiment. Experiments were only performed from under-saturation with respect to the nanoclusters, as U60 nanoclusters do not readily form from aqueous monomeric species under our experimental conditions. That is, the solutions initially did not contain aqueous $\mathrm{U}$, $\mathrm{Li}$, or $\mathrm{K}$; all subsequent measured aqueous $\mathrm{U}, \mathrm{Li}$, and $\mathrm{K}$ came from the dissolution or dissociation of the nanoclusters. After $\mathrm{pH}$ adjustment, the experimental suspensions were capped, placed on a rotator at $40 \mathrm{rpm}$, and allowed to equilibrate for 24 hours before initial sampling.

Each U60 nanocluster suspension was sampled periodically over the course of 12-16 days, and all samples were analyzed for U, Li, and K concentrations. During sampling, the caps of the Teflon reaction vessels were removed to allow air exchange and to ensure that the experimental suspension maintained equilibrium with respect to atmospheric $\mathrm{CO}_{2}$. At each sampling, the $\mathrm{pH}$ was measured and then adjusted if needed with the method described above. Each sample aliquot was initially filtered through a $0.2 \mu \mathrm{m}$ PTFE membrane to test for secondary microscale precipitates but to allow passage of nanoclusters and aqueous species, and then each sample was 
divided into two portions: a portion that was not filtered further for determination of total $\mathrm{U}, \mathrm{Li}$, and K concentrations (termed 'before molecular weight filtration' or 'BMWF'), and a second portion that was further filtered through a $10 \mathrm{k}$ Da molecular weight sieve in order to remove the isolated U60 nanoclusters, enabling the determination of the aqueous concentrations of $\mathrm{U}, \mathrm{Li}$, and $\mathrm{K}$ in each sample (termed 'after molecular weight filtration', or 'AMWF'). Isolated U60 nanoclusters in solution have a mass of $19,891 \mathrm{Da}$, which was calculated from the average nanocluster composition (see discussion of nanocluster composition below). Therefore, the nanoclusters were likely to be removed from suspension by the molecular weight filtration, while smaller aqueous monomeric species would pass through the molecular weight filter (a schematic of the experimental filtration procedure and the portions removed for metals analyses is shown in Figure 2). The effectiveness of the molecular weight filtration in removing isolated U60 nanoclusters was verified with control experiments and electrospray mass spectroscopy (ESIMS; see ESI-MS section for methods). The ESI-MS spectrum of BFMW samples exhibited the three characteristic peaks of U60 nanoclusters centered at approximately 1950, 2150 and 2400 $\mathrm{m} / \mathrm{z}$, while these peaks were absent in the AMWF samples (Figure 3). Using ESI-MS, the nanoclusters were confirmed to be present and stable for the duration of the experiments (Figure $\mathrm{S} 1)$. Additionally, an examination of the lower $\mathrm{M} / \mathrm{Z}$ range indicated that no mid-sized nanoclusters were present in the solutions allowing us to demonstrate that breakdown of the U60 nanoclusters did not occur (Figure S2). Each measured BMWF element concentration represents the sum of the nanocluster concentration of that element and the concentration of aqueous monomeric species of that element that went into solution from the dissolution or dissociation of the nanoclusters. In contrast, the measured AMWF concentrations represent only the concentrations of the aqueous monomeric species that resulted from the dissolution or dissociation of the nanocluster and passed through the molecular weight filters which retain the nanoclusters. Therefore, the difference between these two measured concentrations can be used in order to determine the elemental composition of the nanoclusters in solution.

All experimental samples were acidified and diluted with trace metal grade 5\% nitric acid. Samples were analyzed for U, Li, and K concentrations by inductively coupled plasma optical emission spectroscopy (ICP-OES). Elemental standards were made from commercially available 1,000 ppm standards that were diluted with trace metal grade 5\% nitric acid to match the matrix of the samples. Six standards for each element were prepared for the following concentrations ranges: $10^{-5.59}$ to $10^{-3.42} \mathrm{M}$ for $\mathrm{K}, 10^{-4.80}$ to $10^{-2.70} \mathrm{M}$ for $\mathrm{Li}$, and $10^{-6.34}$ to $10^{-3.64} \mathrm{M}$ for $\mathrm{U}$. U, Li, and $\mathrm{K}$ were analyzed at $766.49 \mathrm{~nm}, 610.362 \mathrm{~nm}$, and $424.167 \mathrm{~nm}$, respectively, and the sets of standards were analyzed at the beginning, middle and end of each ICP-OES run to check for machine drift and analytical uncertainty. Machine drift was negligible and analytical uncertainty was found to be $\pm 0.5 \%$ for $\mathrm{U}, \pm 6.0 \%$ for $\mathrm{Li}$ and $\pm 1.4 \%$ for $\mathrm{K}$.

\subsection{Electrospray Mass Spectroscopy}

The presence of isolated U60 nanoclusters in suspension within the experimental solutions was verified using ESI-MS at the beginning and end of each experiment. A sample was removed from each of the experimental suspensions at the first sampling time, 24 hours after the beginning of the experiment. The other sample was taken during the last sampling for each experimental run. ESI-MS spectra were recorded on a Bruker MicroOTOF-Q II high resolution quadrapole time of flight mass spectrometer apparatus with the following analytical settings: 
$3200 \mathrm{~V}$ capillary voltage, $0.4 \mathrm{Bar}$ nebulizer gas, $4 \mathrm{~L} / \mathrm{min}$ dry gas, $180^{\circ} \mathrm{C}$ dry gas temperature, and negative-ion mode. The samples were introduced by direct infusion at $7 \mu \mathrm{L} / \mathrm{min}$, and scanned over 500-5,000 m/z with data averaged over 2-5 minutes. The results of the ESI-MS measurements are shown in the Supplemental Information section. Under these conditions, the U60 nanoparticles yield a signal of 3 broad peaks (with a width of each of approximately 200 $\mathrm{m} / \mathrm{z}$ ), with the peaks centered at 1965, 2165 and $2450 \mathrm{~m} / \mathrm{z}$. The presence of three peaks is related to the multiple charge states of the U60 nanocluster that are created during the electrospray mass spectrometry analysis due to subtraction of differing amounts of $\mathrm{Li}$ and $\mathrm{K}$ ions from the cluster during the analysis. Multiple peaks are commonly created from single parent species, and they result from the ionization field creating multiply charged ions. The observed broad peaks are caused from cations with different masses being stripped from the parent molecule during ionization, resulting in a range of daughter products with the same charge but slightly different $\mathrm{m} / \mathrm{z}$ ratios. The three peaks are most likely representative of the $-10,-9$ and -8 charge states from left to right, with molecular weights of 19,650, 19,485 and 19,600, respectively. Due to the large size of the U60 nanocluster and the degree of ion-pairing in solution, manual charge state assignment and standard deconvolution algorithms failed. Instead, the MaxEnt algorithm software was used to determine the molecular weight of the U60 molecule. No new peaks were observed at the end of the experiment, which indicates that the U60 nanoclusters were present at the end of the experiments and that no new daughter compounds were created during the course of the experiments.

\subsection{Electrophoretic Mobility and Conductivity}

The electrophoretic mobility measurements of $0.1,0.3,0.5,0.6,1.0,3.0$ and $6.0 \mathrm{~g} / \mathrm{L}$ U60 solutions at different $\mathrm{pHs}$ were conducted using a Malvern Nano-ZS90 zeta potential analyzer at room temperature. The sample solutions were prepared by diluting a concentrated U60 stock solution with deionized water, and were placed in Malvern DTS1060 disposable capillary cells. The solution $\mathrm{pHs}$ were adjusted with concentrated $\mathrm{NaOH}$ or $\mathrm{HCl}$ solutions. The mobility and conductivity were measured under the zeta potential mode with the following settings: $25^{\circ} \mathrm{C}$, 120 s equilibrium time, Hückel model, and an automatically chosen conducting voltage.

\section{Results}

\subsection{Batch Experiments}

The measured BMWF concentrations of $\mathrm{U}, \mathrm{Li}$, and $\mathrm{K}$ exhibited little change between days 1 and 15 as shown in Figure 4 A (see Figures S3, S4, and S5 for the complete dataset). The lack of time dependence for these concentrations suggests that nanocluster dissolution or dissociation occurs rapidly, and that no secondary solid phases precipitated from solution during the course of the experiment. Any solid precipitate likely would have been removed by the initial $0.2 \mu \mathrm{m}$ filtration step of the sample treatment and the BMWF concentrations would have shown a decrease as a function of time.

The measured AMWF Li, and K concentrations each attained a steady-state within $24 \mathrm{hrs,} \mathrm{and}$ these steady-state concentration values were maintained without significant or consistent drift for the duration of the experiment. The AMWF U concentrations decreased slightly over the first 7 
days of the experiments before stabilizing and showing no consistent trend over the final 7 days (Figures 4 B, S6, S7, and S8). The presence of not only Li and K but also structural $\mathrm{U}$ in the AMWF samples suggests the breakdown of whole nanoclusters, and is not consistent with $\mathrm{Li}$ and $\mathrm{K}$ dissociation alone from the nanoclusters or cation exchange. We attribute the small perturbations in the AMWF concentrations over the course of the experiment to a combination of analytical and experimental uncertainties, with the experimental uncertainties arising from small differences in $\mathrm{pH}$ between samples, and from the large dilutions required for ICP-OES analysis. The ratio of partitioning between the aqueous monomeric uranium and total uranium does not depend on the nanocluster concentration; the nanoclusters were found to represent $97.4 \%$ of the total uranium in each of our experiments with a deviation of $0.6 \%$.

Although the measured AMWF U, Li and K concentrations vary by approximately an order of magnitude over the $\mathrm{pH}$ range tested, the concentrations do not fluctuate systematically as a function of $\mathrm{pH}$ (Figure $5 \mathrm{~A}, \mathrm{~B}$ and $\mathrm{C}$ ). In contrast, a clear and well-defined trend in the measured AMWF U, Li and $\mathrm{K}$ concentrations were observed as a function of nanocluster concentration. The AMWF concentrations of $\mathrm{U}, \mathrm{Li}$, and $\mathrm{K}$ each increased with increasing nanocluster concentration with correlation coefficients or $\mathrm{R}^{2}$ values of $0.71,0.70$ and 0.70 , respectively (Figure $6 \mathrm{~A}, \mathrm{~B}$ and $\mathrm{C}$ ). This behavior clearly indicates that isolated nanoclusters in solution do not behave as a bulk solid phase. If they did behave as a solid phase, then the AMWF U, Li and $\mathrm{K}$ concentrations would be buffered by the nanoclusters, and would not exhibit a dependence on the nanocluster concentration in the experimental systems. There was excess U60 nanocluster in each of these experiments; that is, we observed U60 nanoclusters to be present in all of the experimental systems at the time of the last sampling. Hence, the observed increase in AMWF U, $\mathrm{Li}$ and $\mathrm{K}$ concentration with increasing nanocluster concentration was not caused by complete dissolution or dissociation of the nanoclusters in each experiment. Rather, the nanoclusters appear to behave more like aqueous complexes, with higher concentrations of aqueous $\mathrm{U}, \mathrm{Li}$ and $\mathrm{K}$ species in steady-state with higher concentrations of nanoclusters. This suggests that there is at least a steady-state achieved between the isolated nanoclusters in solution and the aqueous monomeric $\mathrm{U}, \mathrm{Li}$ and $\mathrm{K}$ species when equilibrium is approached from the point of undersaturation with respect to the nanocluster.

\subsection{Ion Activity Product Calculations}

If the reaction that describes the U60 nanocluster dissolution or dissociation into component aqueous species is expressed correctly, and if the nanoclusters are in equilibrium with the component aqueous species in the experimental systems, then calculated ion activity product (I.A.P.) values based on the experimental measurements should not vary as a function of $\mathrm{pH}$ or nanocluster concentration. Our calculations of the I.A.P. included two unknowns: 1) the nature and behavior of the nanocluster phase, and 2) the reaction stoichiometry. Solid phases exhibit solubilities that are independent of the concentration of the solid in the system, and hence the activity of a solid is taken to be equal to one if the solid is present as a pure phase. Conversely, aqueous complexes exhibit non-unit activities because their activities vary in direct proportion to the activities of their component aqueous species. Therefore, we use the measurements from our batch experiments as a test of the nature and behavior of the U60 nanoclusters. We calculate I.A.P. values in two ways: 1) considering the nanoclusters as a bulk solid phase with an activity of one, and 2) considering the nanoclusters as an aqueous complex with activities equal to their 
concentration in solution. By determining which approach yields the lowest $\mathrm{pH}$ and nanocluster concentration dependencies for the calculated I.A.P. values, we can determine whether the U60 nanocluster behavior is closer to a bulk solid phase or to an aqueous complex. In addition, we use the data to provide constraints on the equilibrium constant which can be used to make an initial estimate the value of the equilibrium constant for the reaction.

The composition of isolated U60 nanoclusters in solution is not necessarily the same as the stoichiometric composition of the bulk U60 crystal as determined by Sigmon et al. (2009b). The dissolution of the U60 crystal, which consists of U60 nanoclusters and counterions, to isolated U60 nanoclusters in solution may be accompanied by preferential cation leaching, desorption, and/or substitution. The composition of the U60 crystal is defined by the measured BMWF U, $\mathrm{Li}$, and $\mathrm{K}$ molal ratios, assuming that the crystal dissolves completely and that the peroxide and hydroxide molal ratios in the U60 crystal (which cannot be measured) are both equal to the measured molal $U$ composition of the crystal. That is, one peroxide and one hydroxide molecule must be associated with each uranium atom in the crystal structure (Sigmon et al., 2009a). Based on the average of the BMWF measurements from all experiments, the calculated composition of the $\mathrm{U} 60$ crystal is $\mathrm{K}_{16} \mathrm{Li}_{44}\left[\mathrm{UO}_{2}\left(\mathrm{O}_{2}\right) \mathrm{OH}\right]_{60}$, neglecting water of crystallization.

Because the molecular weight filtration step in our sampling procedure removes only the U60 nanoclusters and their intimately associated counterions from suspension, we estimate the stoichiometry of the isolated U60 nanoclusters in solution as the average difference over all experimental measurements between the BMWF U, Li and $\mathrm{K}$ concentrations and the corresponding AMWF concentrations. Since we were unable to directly measure the concentration of free peroxide, we assumed that the peroxide and hydroxide stoichiometries in the isolated U60 nanocluster in solution are both equal to the measured molal U composition of the nanocluster. The resulting average calculated composition of the isolated U60 nanocluster in solution is $\mathrm{K}_{16} \mathrm{Li}_{25}\left[\mathrm{UO}_{2}\left(\mathrm{O}_{2}\right) \mathrm{OH}\right]_{60}$. The $\mathrm{U} 60$ crystal and isolated nanoclusters in solution exhibit identical $\mathrm{K}: \mathrm{U}$ molal ratios, indicating that $\mathrm{K}$ does not leach out of the isolated nanoclusters in solution. However, the Li:U ratio is significantly lower in the isolated nanoclusters in solution than in the crystal, indicating Li leaching when the nanoclusters are suspended in solution. The number of $\mathrm{K}$ per nanocluster was found to have a standard deviation of 1, whereas the Li per nanocluster value had a standard deviation of 4 . The difference between the U60 crystal and the isolated nanocluster Li concentrations indicates that approximately $40 \%$ of the Li leaches into solution as the U60 crystal dissolves to isolated nanoclusters.

The leaching of the $\mathrm{Li}$ is a rapid reaction and reaches steady-state by the first sampling time, as the Li AMWF concentrations showed no time dependency. The leaching of $19 \mathrm{Li}$ from each nanocluster leaves the nanocluster with a charge imbalance and a net charge of $-19 \pm 4$ in solution. Our measured stoichiometry of the isolated nanoclusters in solution, $\left(\mathrm{K}_{16} \mathrm{Li}_{25}\left[\mathrm{UO}_{2}\left(\mathrm{O}_{2}\right) \mathrm{OH}\right]_{60}\right)^{-19}$, is consistent with the determinations by Sigmon et al. (2009b) who concluded that $18 \mathrm{Li}$ atoms in U60 nanocluster crystals are present exterior to the spherical U60 cage structure, with the remaining Li and $\mathrm{K}$ cations present as interior cations. Upon dissolution, the more loosely held exterior cations appear to leach into solution much more readily than the tightly held interior cations. Additionally the order of cation leaching from the U60 nanocluster 
appears to correlate with the charge density of the leaching cation. Li has a larger hydrated radius than $\mathrm{K}$ and therefore a lower charge density, making Li less tightly bound to the nanocluster.

Electrophoretic mobility measurements of the U60 nanoclusters in solution, with values ranging from -3.0 to $-4.7 \mu \mathrm{m}^{*} \mathrm{~cm} / \mathrm{V}^{*} \mathrm{~s}$ for both the 1 and $3 \mathrm{~g} / \mathrm{L}$ nanocluster suspensions (Figure 7 ), qualitatively confirm that the U60 nanocluster is highly negatively charged in solution. The electrophoretic mobility measurements do not vary systematically over the $\mathrm{pH}$ range of this study, suggesting that protonation of the nanoclusters does not occur over this $\mathrm{pH}$ range. A charge of -19 is not unreasonable for the $2.4 \mathrm{~nm}$ diameter nanocluster. For comparison, Zhou and Gunter (1992) found that kaolinite clay particles with a diameter of $200 \mathrm{~nm}$ have a surface charge of $30-60 \mathrm{mC} / \mathrm{m}^{2}$, which is approximately an order of magnitude less than the $159 \mathrm{mC} / \mathrm{m}^{2}$ calculated for U60 nanoclusters. However, the typical specific surface area for a kaolinite sample, 12-24 $\mathrm{m}^{2} / \mathrm{g}$, (Schroth and Sposito, 1997) is also approximately an order of magnitude less than the $548 \mathrm{~m}^{2} / \mathrm{g}$ value calculated for U60. Therefore, both the clay sample and the U60 nanoclusters exhibit roughly similar surface area normalized surface charge.

The conductivity measurements recorded during the electrophoretic mobility measurements can be used to estimate the effective charge of the U60 nanoclusters in solution (SI Table S4). The effective charge is calculated from the sum of the conductivity contributions of all the charged species (including the U60 nanoclusters) in solution. In the case of U60 in water, the total conductance is equal to the sum of the contributions of $\mathrm{H}^{+}, \mathrm{OH}^{-}, \mathrm{Li}^{+}, \mathrm{K}^{+}$, and the U60 nanoclusters. Details of the effective charge calculations are shown in the Supplemental Information. The calculated effective charge, using the Hückel equation to determine the equivalence molar conductivity (EMC) of the U60 nanoclusters from the EMC of the uranyl ion, was found to vary as a function of nanocluster concentration (Figure 8). Over the nanocluster concentration range of $0.1-6.0 \mathrm{~g} / \mathrm{L}$, the calculated charge using this approach varies from -52 to -26. In contrast, the effective charge determined from the measured extent of Li leaching in our experiments (see above discussion) indicates a U60 nanocluster charge of -19, and does not vary as a function of the nanocluster concentration (Figure 8). Determination of the effective charge of the nanocluster from the measured extent of Li leaching is a more direct measure of nanocluster charge than the conductivity measurements because of the reliance of the latter approach on unverified estimations of the EMC for U60 molecules. Therefore, we believe that the Li leaching measurements yield a more accurate estimation of the nanocluster's effective charge, and we use an overall charge of -19 for the nanocluster in all subsequent calculations. The inconsistency between the calculated effective charge of the nanoclusters from the conductivity measurements and that determined from the elemental analyses of the solutions suggests that the use of the Hückel equation for the determination of EMC of macro-ions such as the U60 nanocluster from smaller ions may not be valid (see the Supplemental Information).

For the purpose of calculating I.A.P. values we write a simplified formula for the isolated U60 nanoclusters in solution based on a single uranyl peroxide hydroxide unit while maintaining the same stoichiometric ratios, yielding a composition of: $\mathrm{K}_{0.27} \mathrm{Li}_{0.42}\left[\mathrm{UO}_{2}\left(\mathrm{O}_{2}\right) \mathrm{OH}\right]^{-0.3}$. Using the simplified stoichiometry, the reaction between the isolated nanoclusters and their constituent aqueous species can be represented as:

$$
3 \mathrm{H}^{+}+\mathrm{K}_{0.27} \mathrm{Li}_{0.42}\left[\mathrm{UO}_{2}\left(\mathrm{O}_{2}\right) \mathrm{OH}\right]^{-0.3} \rightarrow 0.27 \mathrm{~K}^{+}+0.42 \mathrm{Li}^{+}+\mathrm{UO}_{2}^{2+}+\mathrm{H}_{2} \mathrm{O}_{2}+\mathrm{H}_{2} \mathrm{O}
$$


with the I.A.P. mass action equation represented as:

I. A. P. $=\frac{a_{\mathrm{K}^{+}}^{0.27} \cdot a_{\mathrm{Li}^{+}}^{0.42} \cdot a_{\mathrm{UO}_{2}+2} \cdot a_{\mathrm{H}_{2} O_{2}}}{a_{\mathrm{H}^{+}}^{3.0} \cdot a_{\mathrm{U} 60 \text { Nanocluster }}}$

where $a$ represents the thermodynamic activity of the subscripted species. In these calculations, activity coefficients for aqueous ions were calculated using the extended Debye-Hückel equation with $\mathrm{A}=0.5105, \mathrm{~B}=0.3285, \stackrel{\mathrm{a}}{=}=5.22$ and $\mathrm{b}=0.02$ (Helgeson et al., 1981). Since we did not buffer ionic strength in these experiments with an electrolyte, and values of a and b have not been determined for uranyl-dominated systems, $\mathrm{RbNO}_{3}$ was chosen as the most reasonable approximation for these experimental solutions, based on cation size, of those for which extended Debye-Hückel parameters are calculated. The ionic strength values that were used to determine activity coefficients were calculated neglecting the contribution from the nanoclusters under the assumption that the highly charged, large nanoclusters cannot be treated as point charges as required by Debye-Hückel theory (Liu, 2010; Haso et al., 2013). Ionic strength in our experimental systems varied from 0.0009 to $0.0060 \mathrm{M}$ as a function of the nanocluster concentration. In a given experiment the ionic strength was found to vary 0.0026 to 0.0031 as a function of $\mathrm{pH}$ and with additions for $\mathrm{pH}$ adjustment. The standard-states that were used for solids phases and $\mathrm{H}_{2} \mathrm{O}$ are the pure mineral or fluid, respectively, at the pressure and temperature of interest. For aqueous species, the standard-state is defined as a hypothetical one molal solution which behaves as if it is infinitely dilute. Activity coefficients of neutral aqueous species are assumed to be equal to one in the calculations.

We calculated I.A.P. values using two approaches: 1) assuming the activity of the nanoclusters is one in order to test if the nanoclusters behave as a bulk solid phase; and 2) assigning a non-unity activity to the nanocluster and assuming their activity to be equal to their concentration in solution. Activities of the aqueous species in equation (2) were calculated from measured total aqueous elemental (AMWF) concentrations in solution by solving a system of nonlinear mass action equations (Table S4) and mass balance equations using a Newton-Raphson iteration program in order to account for the aqueous speciation of each element. An I.A.P. value was calculated for every sampling point in each experiment, and the average for each of the 19 experiments is reported. The mass totals for the mass balance equations for aqueous $\mathrm{U}, \mathrm{Li}$ and $\mathrm{K}$ were determined from the AMWF values measured at each sampling point. The aqueous activity of $\mathrm{H}_{2} \mathrm{O}_{2}$ was determined as a part of the speciation calculations and was based on the assumption that the total concentration of all of the peroxide species in solution was equal to the total concentration of all aqueous $U$ species because, for every mole of $U$ liberated from the nanoclusters, a mole of peroxide is also liberated. Peroxide is miscible in water and does not exsolve from the suspension at the concentrations in the experiments. We assumed that there is negligible reduction of the peroxide due to the short duration of the experiments and because there were no species present in the experimental systems that are readily oxidized. If the peroxide was unstable and a significant portion of the peroxide was reduced during the experiment, then we would expect the dissociation reaction of the U60 nanocluster to be driven to the side of the reactants continually over the course of the experiments. The solutions would not reach equilibrium or steady state with respect to dissolved $\mathrm{U}, \mathrm{K}$, or Li. As the AMWF concentration of the $\mathrm{U}, \mathrm{K}$ and $\mathrm{Li}$ are constant, the solution appears to reach a steady state 
between the aqueous ions and the nanocluster, indicating that peroxide is sufficiently stable over the duration of our experiments. The nanocluster concentration for speciation calculations was calculated from the difference between the measured BMWF and AMWF U concentration, which is equal to the difference between the total element and aqueous concentration. This value was then used with the known nanocluster stoichiometry to calculate the nanocluster concentration. The total $\mathrm{Na}$ and nitrate concentrations added during $\mathrm{pH}$ adjustment of each experiment were also accounted for in the speciation calculations. The experiments were all open to the atmosphere, so we assumed that the concentration of total dissolved carbonate in the experiments was in equilibrium with atmospheric $\mathrm{CO}_{2}$.

The $\mathrm{pH}$ dependencies of the calculated I.A.P. values are shown in Figure 9 A. Neither set of calculated I.A.P. values exhibits a significant trend as a function of $\mathrm{pH}$ over the $\mathrm{pH}$ interval studied. However, the I.A.P. values calculated assuming an activity of one for the U60 nanoclusters exhibit significantly more scatter compared to I.A.P values calculated by treating the U60 nanoclusters as aqueous complexes with activities equal to their concentration in solution. The calculated range of $\log$ I.A.P. values calculated with an activity of one varies from 6.2 to 7.8 with a standard deviation of $+0.3 /-0.9(1 \sigma)$ on the log values; the corresponding range for the values calculated with non-unit activity is 8.6 to 9.4 with a standard deviation of $+0.2 /$ $0.3(1 \sigma)$. The dependencies of the calculated I.A.P. values on the nanocluster concentration are shown in Figure 9 B. For both calculation approaches, the calculated I.A.P. values increase with increasing nanocluster concentration. However, the I.A.P. values that were calculated assuming an activity of the nanoclusters of unity yield a significantly larger slope (with a slope value of 0.20 ) than do the values calculated assuming activities equal to aqueous concentrations for the U60 nanoclusters where the calculated values are close to being independent of nanocluster concentration (with a slope value of 0.08 ).

If the reaction is properly characterized and if the experimental systems each attained equilibrium, then the calculated I.A.P. values can be used to constrain the equilibrium constant and should be approximately equivalent to the true equilibrium constant. Therefore, the calculated I.A.P. values would be independent of $\mathrm{pH}$ and nanocluster concentration. Although neither of our calculation approaches yields I.A.P. values that are completely independent of nanocluster concentration, the approach that treats the isolated U60 nanoclusters as aqueous complexes yields I.A.P. values that exhibit significantly less dependence on nanocluster concentration than does the approach that treats the nanoclusters as a bulk solid phase. An initial estimate of the equilibrium constant can be made averaging all the I.A.P. values which are shown in Figure 9 A that were calculated treating the nanocluster with a non-unit activity, yielding a value of $9.2+0.2 /-0.3(1 \sigma)$ for the log equilibrium constant.

\section{Discussion}

The observed increases in AMWF U, Li, and $\mathrm{K}$ concentrations with increasing nanocluster concentration are not likely a size effect. A correlation between particle size, surface free energy and solubility has long been recognized and is described by Ostwald-Freundlich theory (Ostwald 1900; Freundlich 1909). Early studies such as those of Alexanader (1957) and Schindler et al. (1967) found solubility to increase with decreasing particle size for particles with diameters of the hundreds of $\mathrm{nm}$. Current adaptations of the Ostwald-Freundlich equation predict that the 
solubility of particles above approximately $100 \mathrm{~nm}$ is independent of particle size. With particle sizes smaller than $100 \mathrm{~nm}$, solubility increases with decreasing particle size due to surface free energy effects. However, aggregation and agglomeration could substantially alter surface reactivity by changing the reactive surface area of particles (Stumm et al., 1996; Gilbert and Banfield., 2005; Christian et al., 2008). Therefore, the increases in the AMWF concentrations could be explained if particle size, or even effective particle size due to aggregation, of the isolated U60 nanoclusters decreased with increasing nanocluster concentration in the experimental systems. There is no evidence that the U60 nanocluster size changes as a function of nanocluster concentration. If any change in nanocluster size were to occur as a function of nanocluster concentration, then it is most likely that particle size would increase due to increased aggregation or agglomeration with increasing nanocluster concentration in suspension (Liu, 2010). In that case, we would expect to see an increase in the effective diameter of nanocluster particles and hence a decrease in the AMWF concentrations with increasing nanocluster concentration. However, the DLS measurements that we conducted on suspensions with even higher nanocluster concentrations than those used in the rest of this study indicate a monodisperse population of particles with an average diameter in solution of less than $5 \mathrm{~nm}$ (Figure S9). The DLS cannot precisely determine particle size for particles less than $5 \mathrm{~nm}$, however the DLS results do indicate that aggregation of the nanoclusters does not occur, even in highly concentrated suspensions, and hence the results also rule out particle size effects as the cause of the observed trends in AMWF concentrations with increasing nanocluster concentration.

The small dependence on nanocluster concentration that we observed for the I.A.P. values that were calculated with non-unit activities for the nanoclusters likely arises due to inaccuracies introduced by our assumption equating the nanocluster activity to their concentration in solution, meaning that activity coefficient effects were not included for the nanoclusters in the I.A.P. calculations. If we assume that the activity of the U60 nanoclusters is equal to their aqueous concentration, then the resulting calculated I.A.P. values are close to being independent of nanocluster concentration. Improving our understanding of the activity of the isolated nanoclusters in solution may eliminate the small trend in the calculated I.A.P. values as a function of nanocluster concentration. Methods such as the extended Debye-Hückel equation have been used to model the non-ideal behavior of POM clusters with mixed results (Kozik and Baker, 1990; Geletti et al., 2005; Czap et al., 2006). Czap et al. (2006) found that the extended Debye-Hückel equation fails to account for non-ideal aqueous behavior of tungstoaluminate POM clusters when the ion size parameter, å, is set to be the actual ion size, and that only by either fitting an empirical value for å or by adding an empirical correction term can reasonable approximations of the non-ideal behavior of the cluster be achieved. The tungstoaluminate POMs studied by Czap et al. (2006) have significantly smaller charges ( -5 or -6$)$ than the larger U60 nanocluster which has a charge of -19. Using the crystallographic radius of $1.2 \mathrm{~nm}$ for an estimate of å, the activity coefficients calculated for the U60 nanocluster using the extended Debye-Hückel equation dramatically over-correct for the non-ideal behavior of the U60 in solution that we observed. The over-correction suggests that the extended Debye-Hückel equation, which was designed to account for non-ideal behavior of point charges in solution, is not adequate for modeling the non-ideal aqueous behavior of nanoclusters, POMs, and macromolecules with high associated charges. Empirical methods such as using Setchenow coefficients to calculate activity coefficients could be used to approximate the activity of the U60 
nanoclusters in our study, however such empirical approaches would only be valid in systems with the same $\mathrm{pH}$, ionic strength and ion composition as those studied, and a wider range of experimental conditions would be required to place rigorous constraints on these parameters.

There are few previous experimental constraints on the thermodynamic behavior of nanoparticles in this size range. However, our interpretation that the isolated U60 nanoclusters exhibit non-unit activities as do aqueous complexes is consistent with the computational results of Rusanov et al. (2007), who used a theoretical approach to define the chemical potential effects of the particle size effect on the Gibbs equation. Their work concludes that a suspended nanoparticle's behavior is dominated by its surface atoms which make up a disproportionately large fraction of the atoms in a nanoscale particle relative to those in larger particles. Surface free energies can strongly dominate the overall chemical potential of the nanoparticle phase, and hence yield dissolution and precipitation behaviors that differ from those of a macroscopic solid bulk phase.

\section{Conclusions}

In this study, we measured the extent of dissociation of U60 nanoclusters into monomeric aqueous species from under-saturation with respect to the U60 nanoclusters as a function of time, $\mathrm{pH}$ and nanocluster concentration. Our results demonstrate that a steady-state between the nanoclusters and aqueous species is achieved rapidly in the experimental systems and maintained for at least 15 days. The measured aqueous $\mathrm{U}, \mathrm{Li}$, and $\mathrm{K}$ concentrations do not vary consistently as a function of $\mathrm{pH}$ over range studied. However, the AMWF concentrations increase significantly and consistently with increasing nanocluster concentration in the suspension. This observation indicates that the nanoclusters exhibit dissociation behavior characteristic of aqueous complexes rather than of a bulk solid phase. The concentration of an aqueous complex increases as the concentrations of components of the complex increase in the system. Conversely, bulk solid phases buffer the solubility. Thus, if the nanoclusters behaved as a solid phase, their presence would yield measured AMWF U, Li, and $\mathrm{K}$ concentrations that are independent of nanocluster concentration in the system. The reaction behavior of the nanoparticles was further tested by calculating the ion activity product for the dissolution or dissociation reaction. The nanoclusters were treated as either a solid phase with an activity of 1 or a highly charged aqueous complex with an activity equal to the concentration of the nanoclusters. The I.A.P. values calculated using non-unit activities exhibit a significantly lower dependence on nanocluster concentration than do those calculated using a nanocluster activity of one. The results strongly suggest that isolated U60 nanoclusters in solution do not behave as bulk solid phases and do not buffer the AMWF metal concentrations. Our measurements provide initial constraints on the equilibrium constant for the dissociation reaction. Further constraints require a better characterization of the composition, the overall charge of the nanoclusters in aqueous environments and reversal experiments in order to confirm reaction reversibility and to yield a more precise equilibrium constant value. Given that the majority of the uranium within the system is present in the nanoclusters, our results indicate that an understanding of nanocluster stability is essential for determining the mobility of uranium in any system where uranyl nanoclusters may be present.

\section{Acknowledgements}


This material is based upon work supported as part of the Materials Sciences of Actinides Center, an Energy Frontier Research Center funded by the U.S. Department of Energy, Office of Science, Office of Basic Energy Sciences under Award Number DE-SC0001089. ICP-OES analyses were conducted at the Center for Environmental Science and Technology at the University of Notre Dame. Electrospray Mass Spectrometry was performed in the Mass Spectrometry and Proteomics Facility at the University of Notre Dame supported by the National Science Foundation (CHE-0741793). Three journal reviews and suggestions made by editor Marc Norman significantly improved the presentation of the research, and are greatly appreciated.

\section{References}

Antonio M.R., Nyman M., and Anderson T.M. (2009) Direct observation of contact ion-pair formation in aqueous solution. Angwe. Chem. Int.. 48: 6136-6140.

Armstrong C. R., Nyman M., Tatiana S., Sigmon G. E., Burns P. C., and Navrotsky A. (2011) Uranyl peroxide enhanced nuclear fuel corrosion in seawater. PNAS, 109: 1874-1877.

Burns P.C. (2011) Nanoscale uranium-based cage clusters inspired by uranium mineralogy. Mineralogical Magazine, 75: 1-25.

Burns P.C., Ewing R.C. and Navrosky A. (2012), Nuclear fuel in a reactor accident. Science, 335: $1184-1187$.

Burns P.C., Kubatko K.A., Sigmon G., Fryer B. J. Gagnon, J. E. Antonio, M. R., and Soderholm L. (2005), Actinyl Peroxide Nanospheres. Angew. Chem. Int. Ed., 44: 2135-2139.

Christian P., Von der Kammer F., Baalousha M., and Hofmann Th. (2008) Nanoparticles: structure, properties, preparation and behaviour in environmental media. Ecotoxicol., 17: 326343.

Czap A., Neuman N.I., and Swaddle T.W. (2006), Electrochemistry and homogeneous selfexchange kinetics 12-tungstoaluminate (5-/6-) couple. Inorg. Chem., 45, 9518-9530.

Freundlich H. (1909) Kolloidchemie. Akademischer Verlag, Leipzip (English translation: 1923, E.P.Dutton Co. Publ., New York).

Geletti Y.V., Hill C.L., Bailey A.J., Hardcastle K.I., Atalla R.H., and Weistock (2005) Electron exchange between $\alpha$-keggin tungstoaluminates and a well-defined cluster-anion probe studies of electron transfer. Inorg. Chem., 44, 8955-8966.

Gilbert B., and Banfield J.F. (2005) Molecular-scale processes involving nanoparticulate minerals in biogeochemical systems In: Molecular Geomicrobiology (J.F. Banfield, J CerviniSilva and K.M. Nealson, eds.) Reviews in Mineral. 59, 109-155 Mineralogical Society of America, Washington DC. 
577 Guillaumont R., Fanghanel T., Fuger J., Grenthe I., Neck V., Palmer D., and Rand M. (2003)

578 Updated on the Chemical Thermodynamics of Uranium, Neptunium and Plutonium, second ed.

579 Elsevier, Amsterdam.

580 Haso F., Fang X., Yin P., Ross J.L., and Liu T. (2013) The self-assembly of a macroion with

581

582

583

584

585

586

587

588

589

590

591

592

593

594

595

596

597

598

599

600

601

602

603

604

605

606

607

608

609

610 anisotropic surface charge density distribution. Chem. Commun., 49: 609-611.

Haynes W.M. (2013) CRC Handbook of Chemistry and Physics. CRC Press, Boca Raton, FL.

Helgeson H.C., Kirkham D.H. and Flowers G.C. (1981) Theoretical prediction of the thermodynamic behavior of aqueous-electrolytes at high pressures and temperatures. 4 . Calculation of activity-coefficients, osmotic coefficients, and apparent molal and standard and relative partial molal properties to 600-degrees-C and $5 \mathrm{~kb} \mathrm{Am.} \mathrm{J.} \mathrm{Sci.,} \mathrm{281:} \mathrm{1249,1516.}$

Hou Y., Alam T.M., Rodriguez M.H. and Nyman M. (2012) Aqueous compatibility of group IIIA monomers and Nb-polyoxoanions Chem. Commun., 48: 6004-6006.

Hou Y., Nyman M., and Rodriguez M.H. (2011) Soluble heteropolyniobates from the bottom of group IA Angew. Chem. Int., 50: 12514-12517.

Kozik M., and Baker L.C.M. (1990) Electron-exchange reactions between heteropoly anionscomparison of experimental rate constants with theoretically predicted values. Journal of the American Chemical Society, 112, 7604-7611.

Liu, T. Supramolecular (2002) Structures of polyoxomolybdate-based giant molecules in aqueous solution. Journal of the American Chemical Society, 124: 10942-10943.

Liu T., Diemann E., Li H.L., Dress A.W.M., and Muller A. (2003) Self-assembly in aqueous solution of wheel-shaped Mo-154 oxide clusters into vesicles. Nature, 426: 59-62.

Liu, T.B. (2010) Hydophillic Macroionic Solutions: What happens when soluble ions reach the size of nanometer scale? Langmuir, 26: 9202-9213.

Liu G., Kistler M.L., Li T., Bhatt A., and Liu (2006) Counter-ion association effect in dilute giant polyoxometalate $\left[\left(\mathrm{Ass}_{12}^{\mathrm{III}} \mathrm{Ce}^{\mathrm{III}}{ }_{16}\left(\mathrm{H}_{2} \mathrm{O}\right)_{36} \mathrm{~W}_{148} \mathrm{O}_{524}\right]^{76-}\left(\left\{\mathrm{W}_{148}\right\}\right)\right.$ and $\left[\mathrm{Mo}_{132} \mathrm{O}_{372}\left(\mathrm{CH}_{3} \mathrm{COO}\right)_{30}\right.$ $\left.\left(\mathrm{H}_{2} \mathrm{O}\right)_{72}\right]^{42-}\left(\left\{\mathrm{Mo}_{132}\right\}\right)$ macroanionic solutions. Journal of Cluster Science, 17: 427-443.

Martell R. J. and Smith R. M. (2001) NIST Critical selected stability constants of metal complexes, Version 6.0. NIST Standard Reference Database 46. National Institute of Standards and Technology. Gaithersburg, MD.

Miro, P. and Bo, C. (2012) Uranyl-peroxide nanocapsules: electronic structure and cation complexation in $\left[(\mathrm{UO} 2)_{20}\left(\mu-\mathrm{O}_{2}\right) 30\right]^{20-}$ Inorganic Chemistry, 51: 3840-3845.

Muller A., Krogerler P. and Dress A.W.M. (2001) Giant metal-oxide-based spheres and their topology: from pentagonal building blocks to keplerates and unusual spin systems. Coordination Chemistry Reviews, 222: 193-218. 
611 Nyman M. and Burns P.C. (2012) A comprehensive comparison of transition-metal and actinyl 612 polyoxometalates Chemical Society Reviews, 41: 7354-7367.

613 Ostwald W. (1900) Über die vermeintliche isomerie des roten und gelben Quesck-silberoxyds 614 und die Oberflachenspannung fester Körper. Z. Phys. Chem., 34: 495-503.

615 Pigga J.M., Teprovich J.A., Flowers R.A., Antonio M.R. and Liu T. (2010) Selective monovalent 616 cation association and exchange around keplerate polyoxometalate macroanions in dilute 617 aqueous solutions. Langmuir, 26: 9449-9456.

618 Qui, J and Burns P.C. (2013) Clusters of actinides with oxide, peroxide, or hydroxide bridges.

619 Chemical Reviews, DOI: 10.1021/cr300159x.

620 Qui J., Ling, J., Sui, A., Szymanowski, J.E.S., Simonetti, A. and Burns P.C. (2012) Time 621 resolved self-assembly of a fullerene-topology core-shell cluster containing 68 uranyl polyhedra.

622 Journal of the American Chemical Society, 134: 1810-1816.

623 Roy K.I., and Lucy C.A. (2005) Influence of methanol as a buffer additive on the mobilities of 624

625 626

627

628

629

630

631

632

633

634

635

636

637

638

639

640

641

642

643

644

organic cations in capillary electrophoresis. Electroporesis, 24, 370-379

Rusanov A. I., Shchekin A.K. (2007) On the formulation of the material equilibrium condition for a dissolving solid nanoparticle A.I.P., 127: 191102.

Schindler, P.W. (1967) Heterogeneous equilibria involving oxides, hydroxides, carbonates and hydroxide carbonates. In Equilibrium Concepts in Natural Water Systems, Adv. Chem. Ser. No. 67, American Chemical Society, Washington DC, p. 196.

Sheldrick, G. M. (1997) SHELX-97 Program pack or solution and refinement of crystal structures from X-ray diffraction data, Bruker AXS, Inc. Madison, WI.

Schroth B.K., and Sposito G. (1996) Surface charge properties of kaolinite. Clay and Clay Minerals, 45: 85-97.

Sigmon G.E., Ling J., Unruh D.K., Moore-Shay L., Ward M., Weaver B., and Burns P.C. (2009a) Uranyl-peroxide interaction favor nanocluster self-assembly, J.A.C.S., 131: 1664816649.

Sigmon G.E., Unruh D.K., Ling J., Weaver B., Ward M., Pressprich L., Simmonetti A., and Burns P.C. (2009b) Symmetry verses Minnumal Pentagonal Adjacencies in Uranium-Based Polyoxometalate Fullerene Topologies Angew. Chem. Int. Ed., 48: 2737-2740.

Stumm W., and Morgan J.J. (1996) Aquatic Chemistry Chemical Equilibrium Rates in Natural Waters, third ed., John Wiley and Sons, Inc. New York, NY.

Vlaisavljevich B., Gagliardi L. and Burns P. C. (2010) Understanding the structure and formation of uranyl peroxide nanoclusters by quantum chemical calculations, J.A.C.S., 132: 14503-14508. 
645 Zaninato P. L., Di Bernardo P., and Grenthe I. (2012) Chemical equilibria in the binary and 646 ternary uranyl(VI)-hydroxide-peroxide systems. Dalton Transactions, 41: 3380-3386.

647 Zhang J., Wang Y., Liang D., Ying Q., and Chu B. (2005) Association behavior of PDMA-g648 PMMA in mixed solvents and its application as a DNA separation medium. Macromolecules, 38, 649 1936-1943

650 Zhou Z., and Gunter W.D. (1992) The nature of the surface charge of kaolinite. Clays and Clay 651 Minerals, 40: 365-368. 


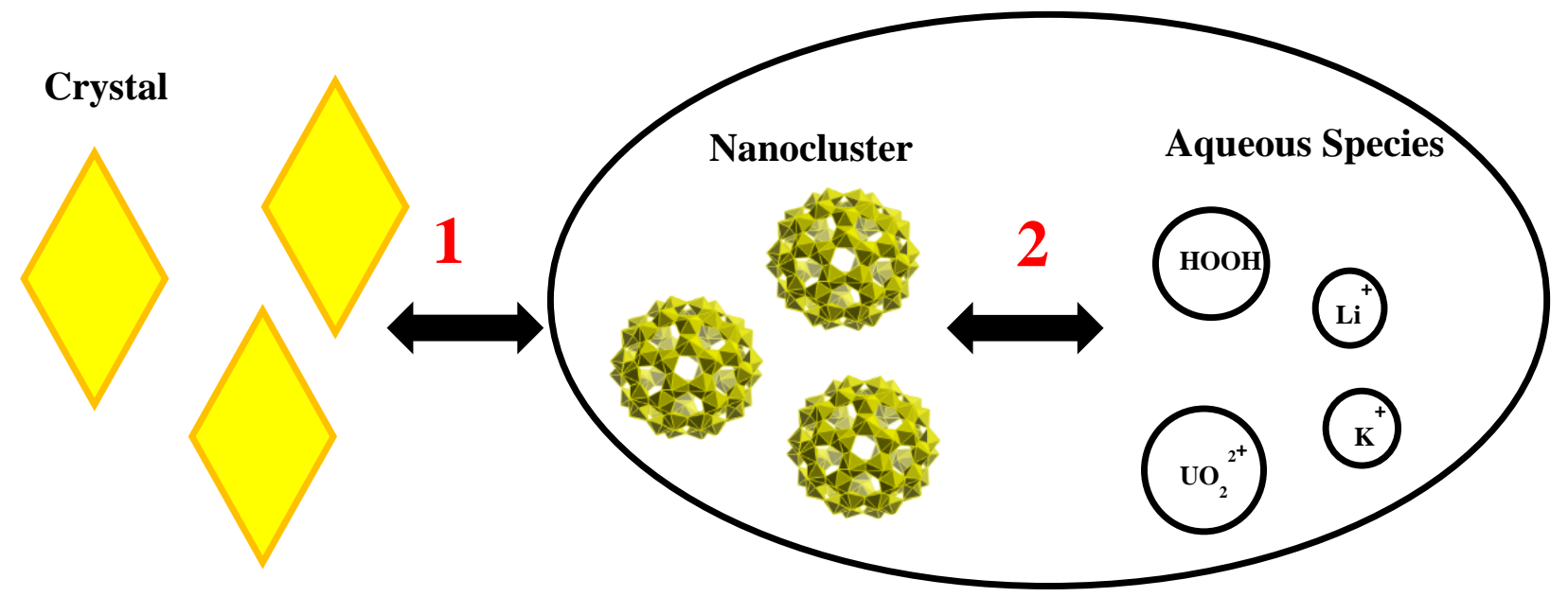

Figure 1. The three potential phases (crystal, nanocluster and monomeric aqueous species) and the two potential equilibria in a nanocluster system. This study focuses on Equilibrium 2 only.
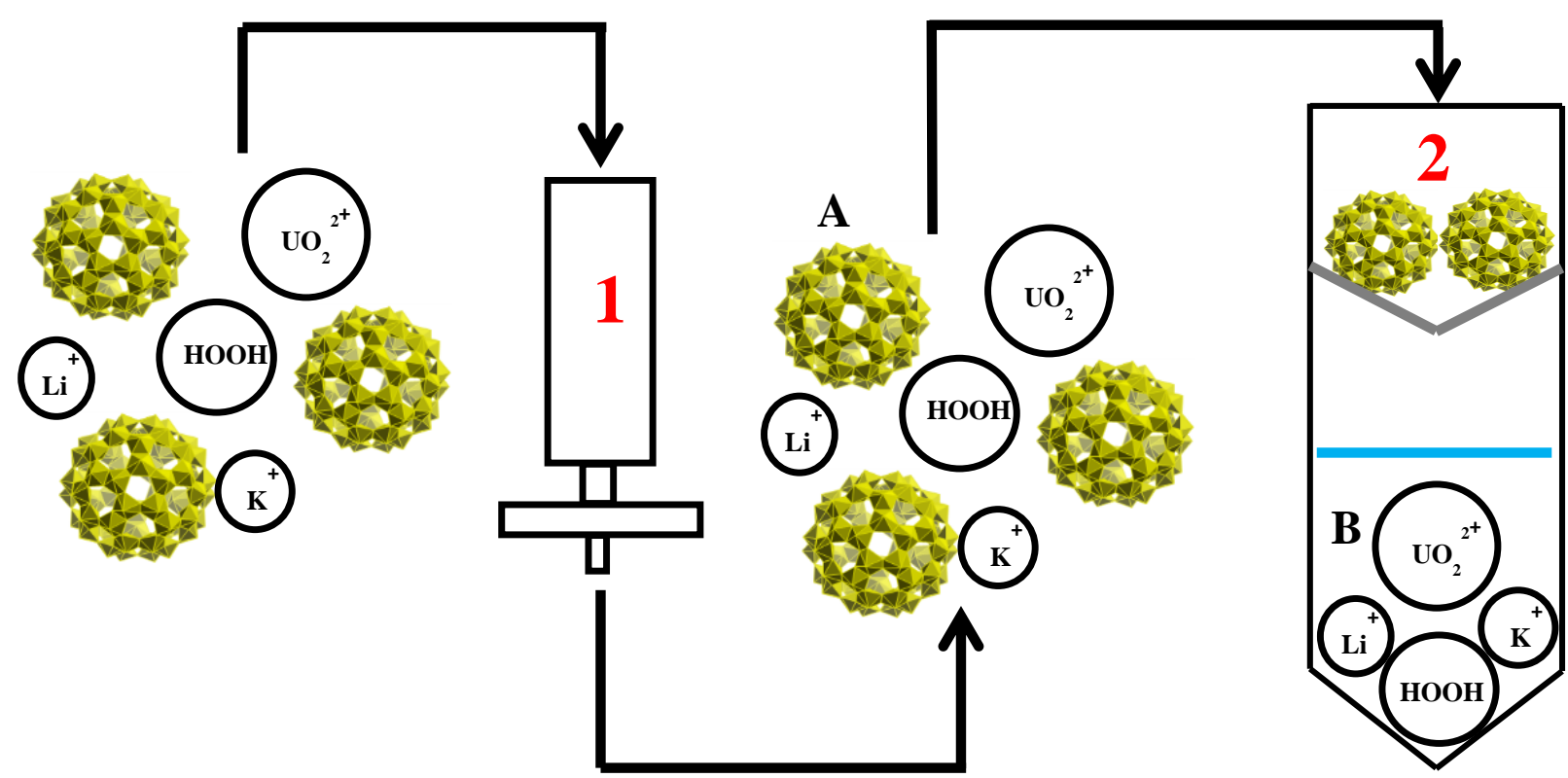

Figure 2. Schematic of experimental filtration process. 1) $0.2 \mu \mathrm{m}$ PTFE membrane used to test for secondary microscale particulates. 2) 10,000 Da molecular weight filter to separate the nanocluster from the monomeric aqueous species. All samples were divided into two portions for the analysis of $\mathrm{U}, \mathrm{Li}$ and $\mathrm{K}$ : (A) Before molecular weight filtration (BMWF) which measured the element concentrations after filtration step 1, but before filtration step 2; and (B) the After molecular weight filtration (AMWF) which measured the element concentrations after filtration step 2. 


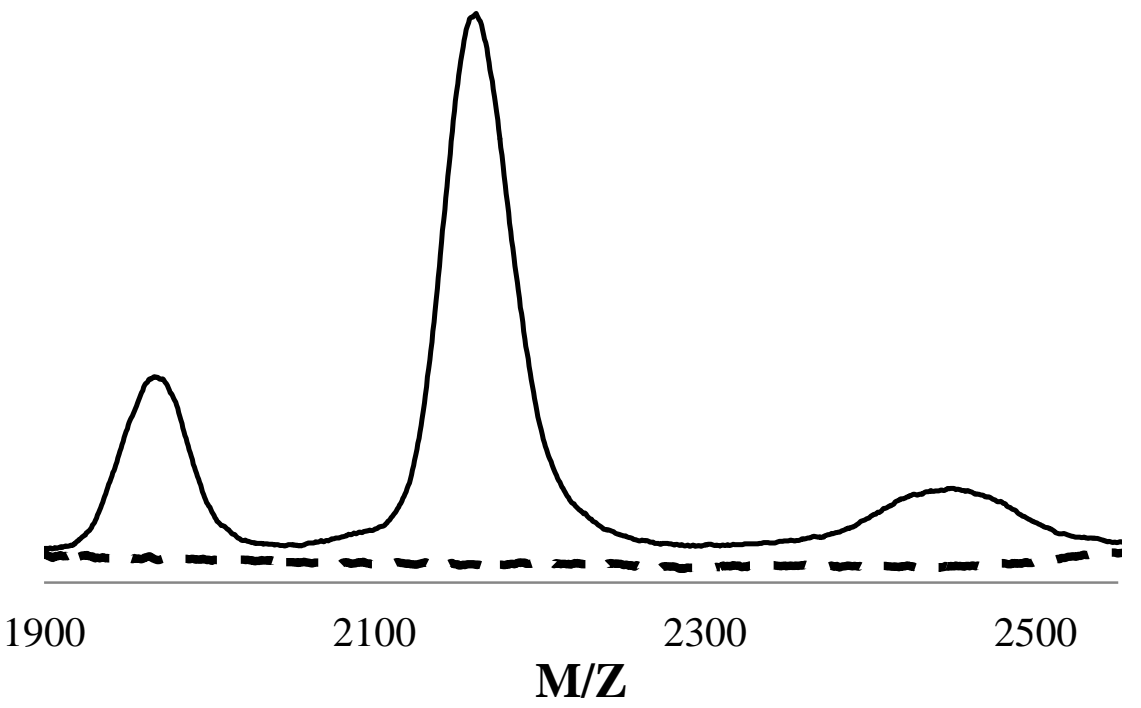

Figure 3. Representative electrospray mass spectroscopy spectra that demonstrate complete removal of U60 by the molecular weight filtration approach used in this study. The spectrum from the before molecular weight filtration (BMWF) sample is represented by the solid curve that displays the characteristic three peak signature of U60; and the spectrum from the after molecular weight filtration (AMWF) spectrum is represented by the dashed curve. 

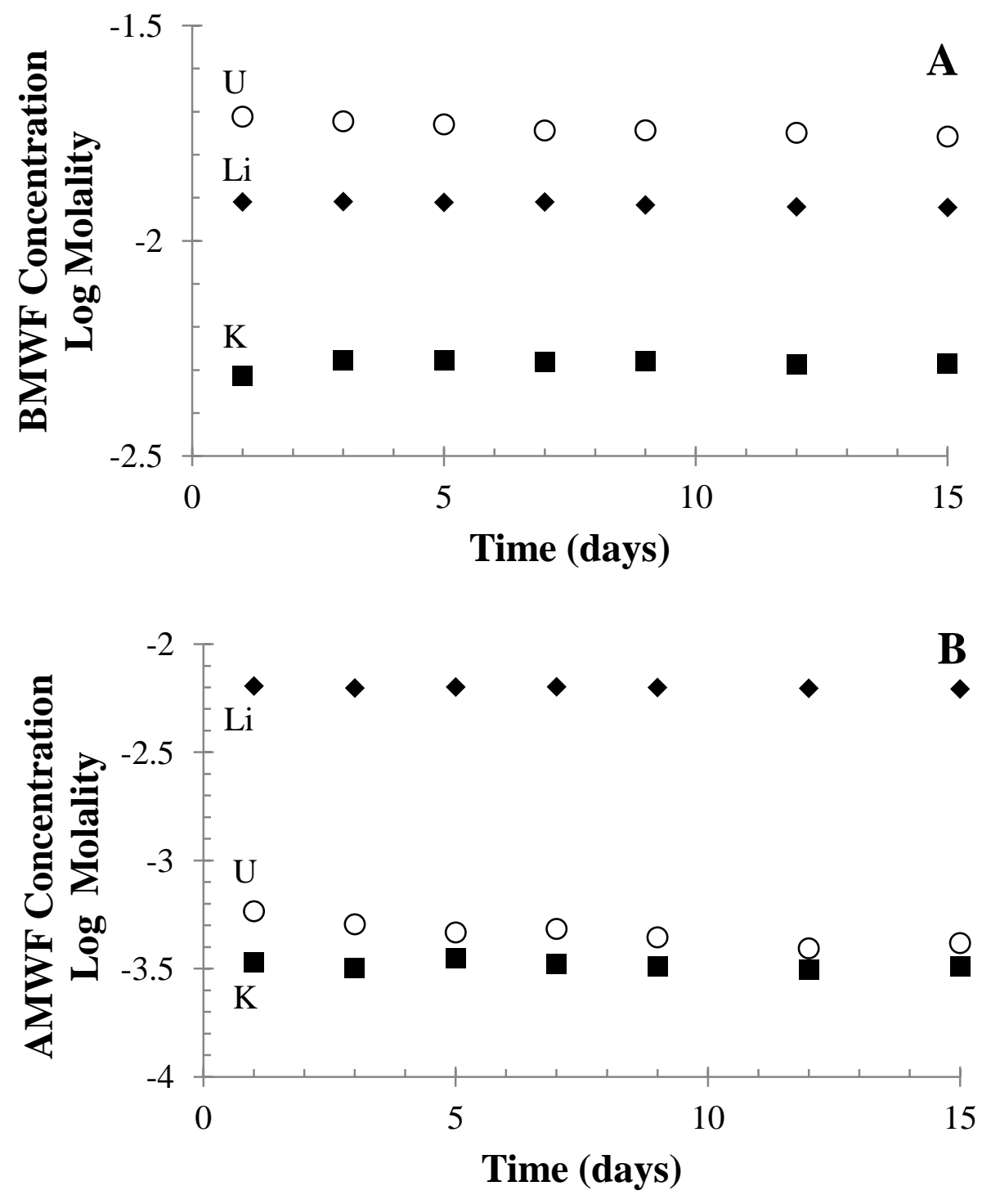

Figure 4. Representative results from stability experiments conducted under the following conditions: $\mathrm{pH} 7.5$ and $5.85 \mathrm{~g} / \mathrm{L}$ U60. Remaining experimental data are shown in the Supplemental Information section. A) Before molecular weight filtration (BMWF) concentrations of $\mathrm{U}, \mathrm{Li}$ and $\mathrm{K}$; B) After molecular weight filtration (AMWF) concentrations of $\mathrm{U}, \mathrm{Li}$ and $\mathrm{K}$. 

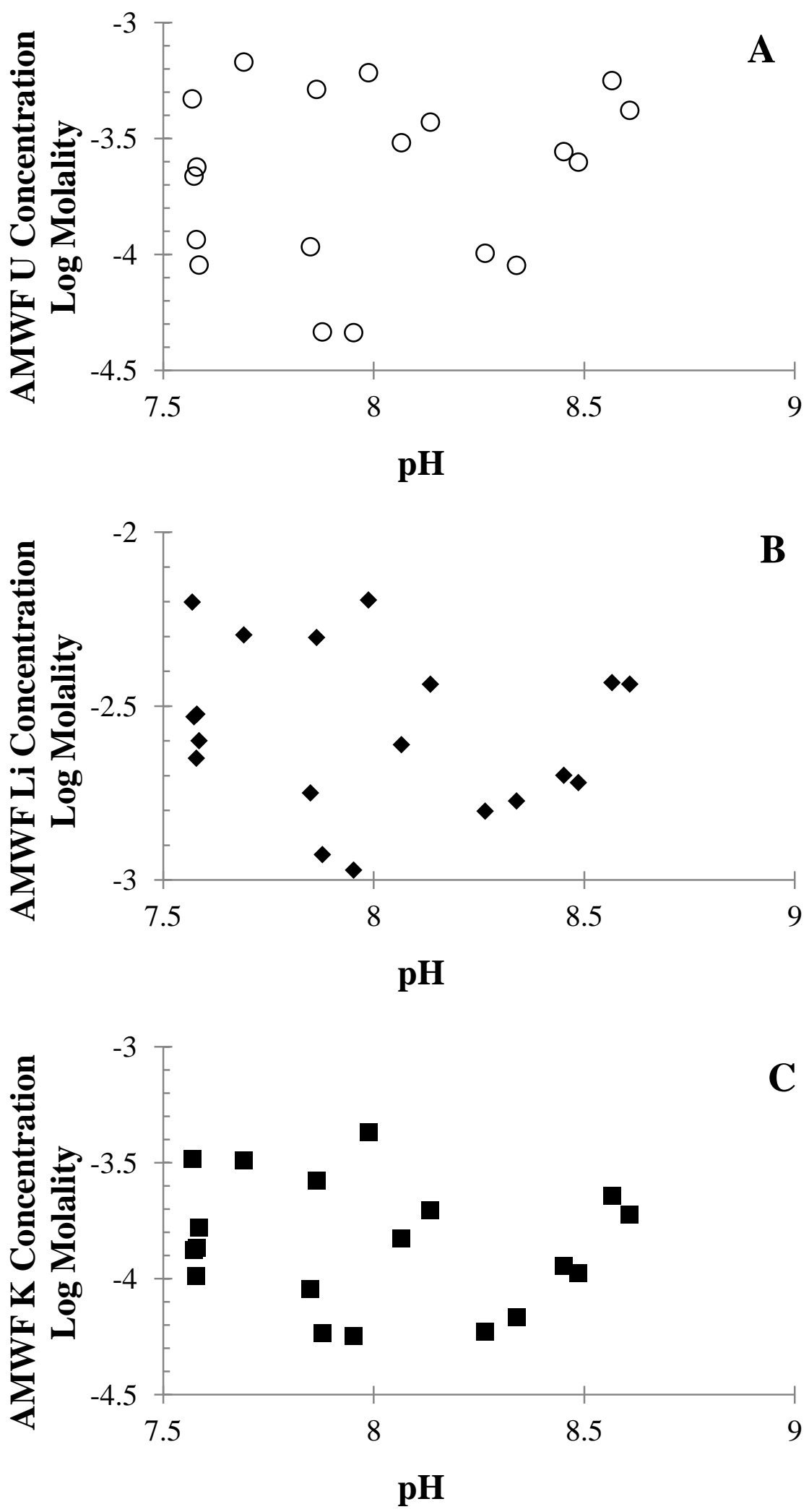

Figure 5. Measured U, Li and K concentrations after molecular weight filtration (AMWF) from all experiments. Each data point represents the average concentration for all samples taken in a particular experiment, the data are plotted as a function of final solution $\mathrm{pH}$ for $\mathrm{U}, \mathrm{Li}$ and $\mathrm{K}$, respectively. 

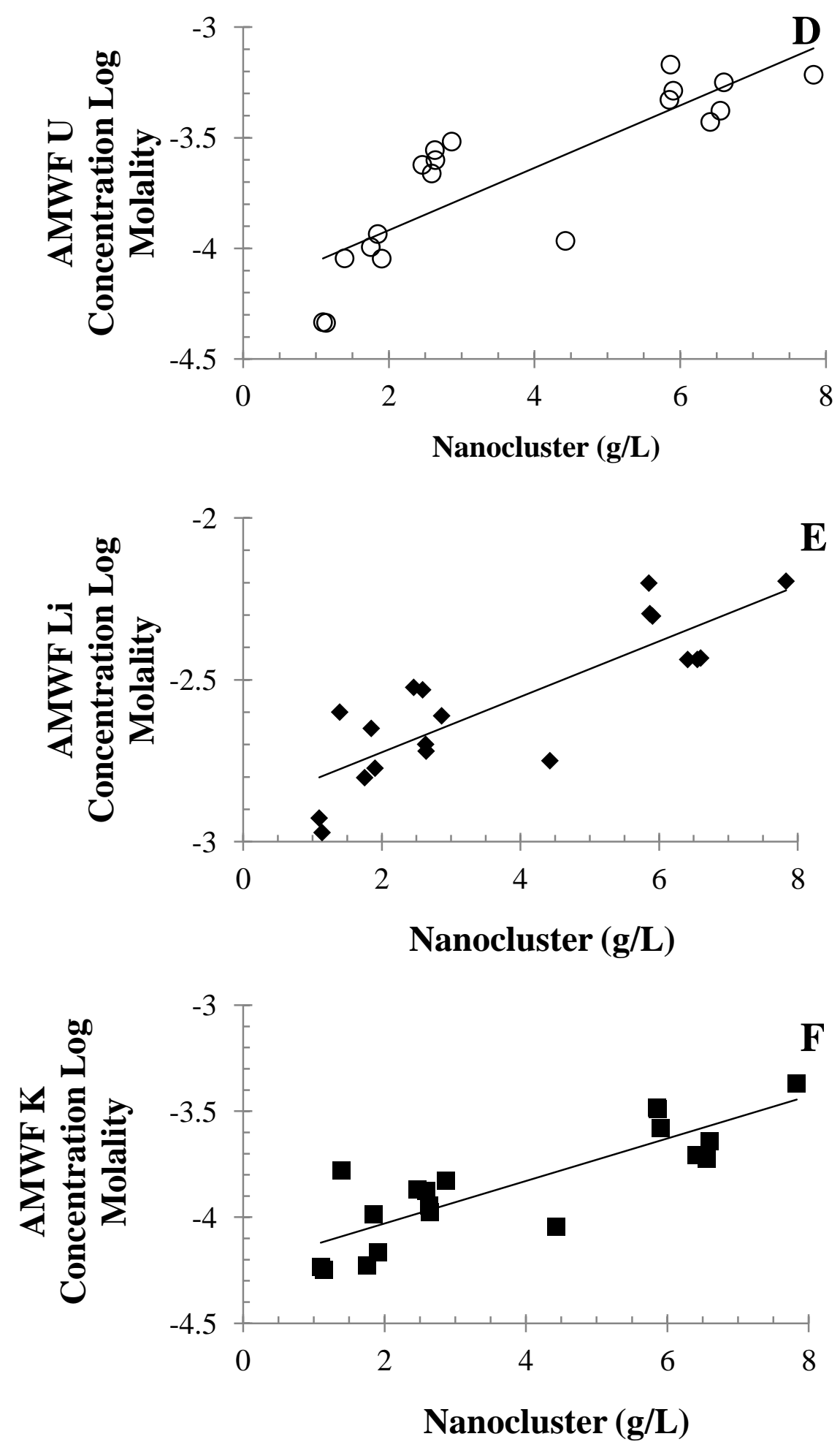

Figure 6. Measured U, Li and K concentrations after molecular weight filtration (AMWF) from all experiments. Each data point represents the average concentration for all samples taken in a particular experiment are plotted as a function of nanocluster concentration in each experiment for $\mathrm{U}, \mathrm{Li}$ and $\mathrm{K}$, respectively. Line are visual aids to show the increase in aqueous $\mathrm{U}, \mathrm{Li}$ and $\mathrm{K}$ with increase nanocluster additions. 


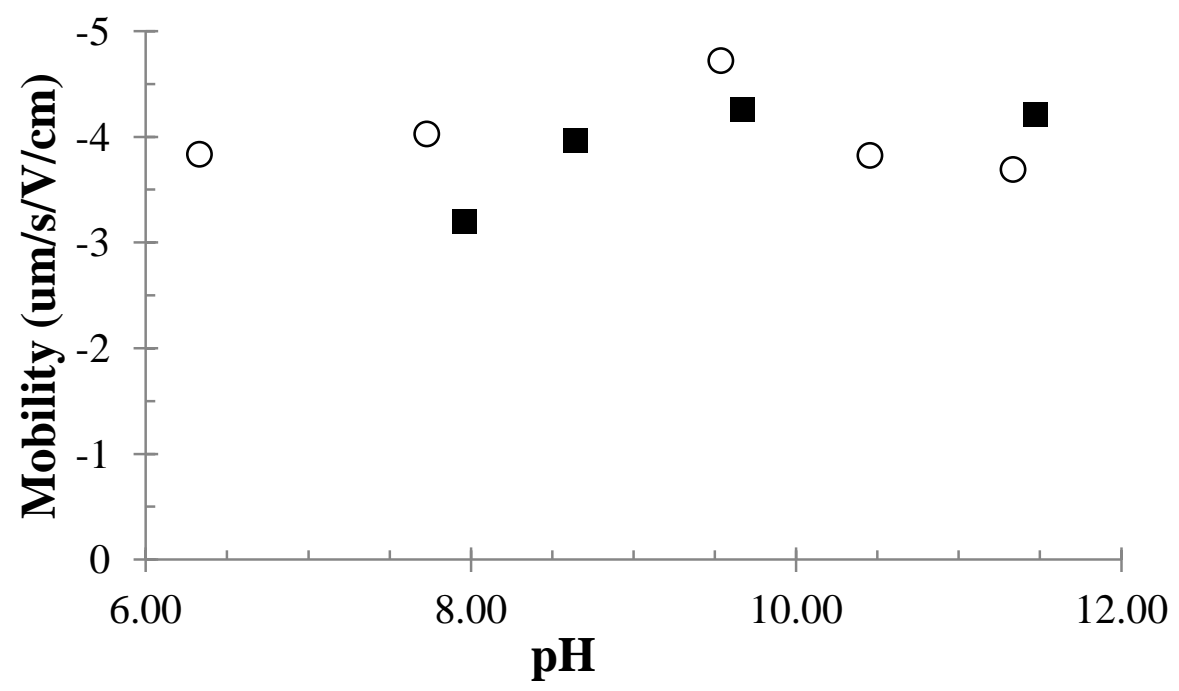

Figure 7. Measured electrophoretic mobility of isolated U60 nanoclusters in solution. The circles represent experiments with $1 \mathrm{~g} / \mathrm{L}$ of $\mathrm{U} 60$, and the squares represent experiments with $3 \mathrm{~g} / \mathrm{L}$ of U60.

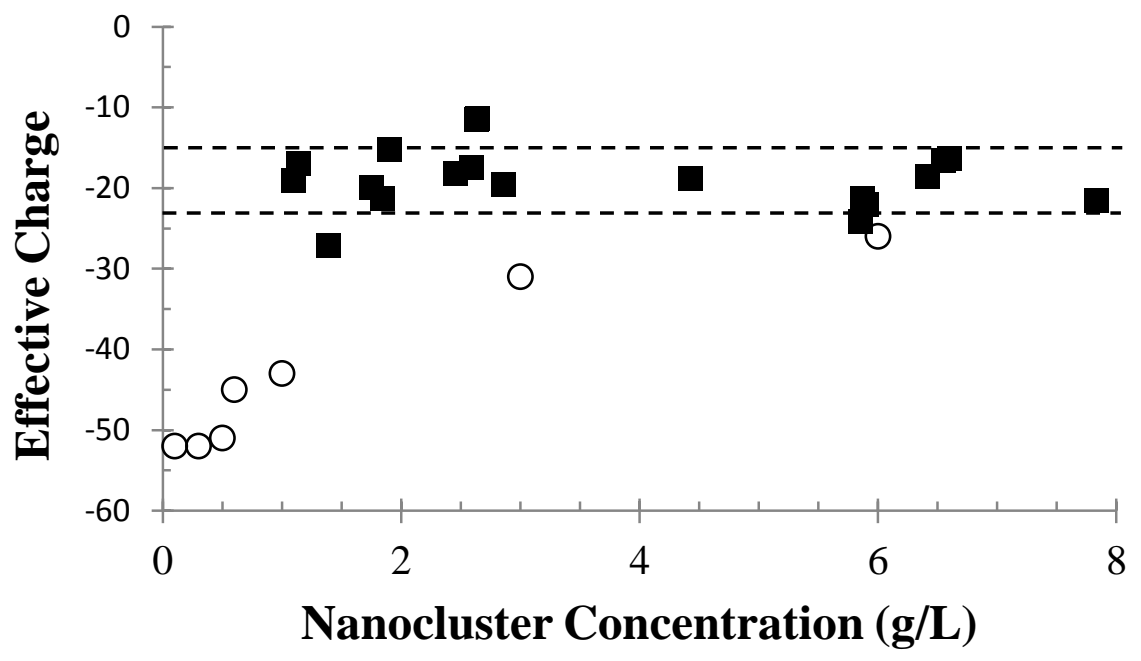

Figure 8. The calculated effective charge of a U60 nanocluster in solution as determined by the nanocluster composition in solution (filled squares) compared to the calculated effective charge calculated from conductivity open circles). The dashed lines represent 1 standard deviation around the mean effective charge value of -19 for the nanocluster composition-based data. 

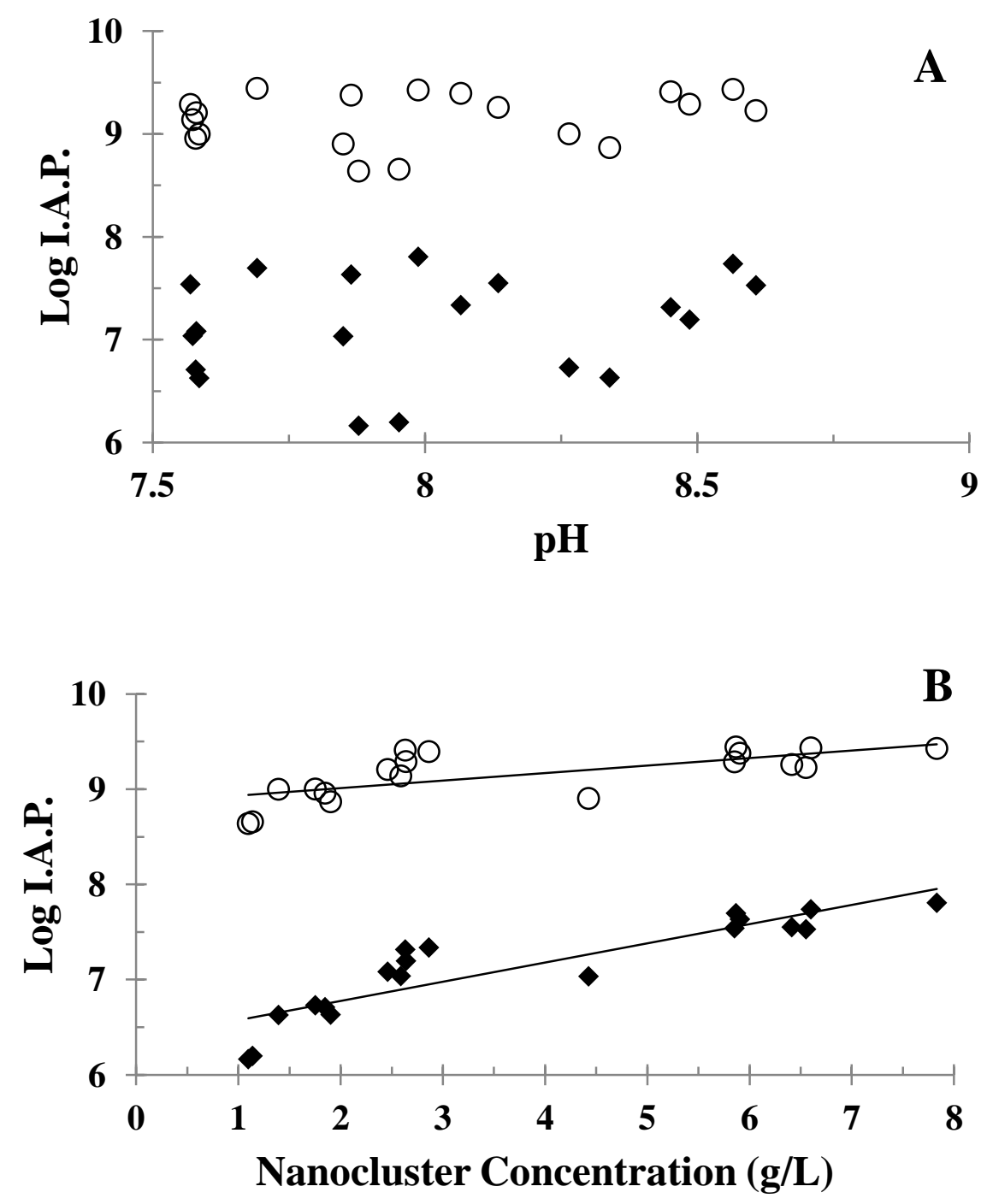

Figure 9. Calculated I.A.P. values, each data point represents the average calculated I.A.P. value for a given experiment. The I.A.P. values that are represented by diamonds were calculated by treating the nanoclusters as a solid phase with an activity of one. The circles represent the I.A.P. values calculated by treating the nanoclusters as aqueous complexes with an activity equal to the nanocluster concentration in solution. A) I.A.P. values as a function of $\mathrm{pH}$; B) I.A.P. values as a function of nanocluster concentration. Lines are linear best-fits of each set of values. 